\title{
Two helpful developments towards better understanding of adiabatic invariance in classical mechanics
}

\author{
J. R. Mohallem*10 \\ ${ }^{1}$ Laboratório de Átomos e Moléculas Especiais, Departamento de Física, ICEx, Universidade Federal de Minas Gerais, PO Box \\ 702, 30123-970, Belo Horizonte, MG, Brasil
}

Received on July 17, 2018; Revised on July 31, 2018; Accepted on August 20, 2018.

\begin{abstract}
The topic of difficult understanding of adiabatic invariance in classical mechanics is dealt with in a more understandable way. Using the one-dimensional harmonic oscillator as an example, the goals of this paper are twofold. First, given a first-order parameter variation, the second-order magnitude of the correction to the adiabatic invariant is established in simple terms. Second, the identification of the action variable with the invariant quantity for slow variations of different parameters of the Hamiltonian is confirmed, by invoking the correct equation of motion in the derivation.
\end{abstract}

Keywords: Adiabatic invariant, classical mechanics.

\section{Introduction}

Aside from ideal isolated systems, the common conservation laws do not usually hold in the physics of real systems. In consequence, quasi-invariant quantities start playing an important role in the approximate assessment of many physics problems involving oscillations. Talman quotes adiabatic invariance as perhaps the most essential, as well as the least understood, concept in acelerator physics [1]. I would say further, one of the least understood concepts of fundamental physics. It's never too much to recall the role played by adiabatic invariants in quantum mechanics [2], so that efforts to clarify their meaning should be welcome.

It seems amazing that, in spite of this, the subject of adiabatic invariance is treated only shortly in classical mechanics textbooks, quoting Devaud et al. 3], almost as "but a curiosity". In order to introduce the concept, most authors restrict themselves to present some variation of Einstein's proof in 1911 that the action variables $J_{k}$ of a multi-periodic mechanical system are the adiabatic invariants $4[6]$. In many years teaching classical mechanics for undergraduate and graduate courses, I noted that the fate of $J_{k}$ of being an adiabatic invariant is almost useless to help students to understand well the concept. Furthermore, this approach restricts the subject to advanced mechanics courses, since it demands knowledge of Hamilton-Jacobi theory. In fact, most undergraduate textbooks do not even refer to it 7,9 . The alternative procedure, namely to replace fast oscillating terms by their average values, in the expressions for time derivatives of the energy [10], requires subtle and smart reasoning that frequently yields wrong results.

${ }^{*}$ Correspondence email address: rachid@fisica.ufmg.br
"What is really an adiabatic invariant" remains as a challenging question for students and even for lecturers.

The theme appears more frequently in teaching papers. A derivation of adiabatic invariance from Rund-Trautman identity and Noether's theorem [11] is very interesting but seems to be as inextricable for beginner students as the derivation via the action variables. Some elementary examples are treated in 12 21. Crawford explores the elementary and $J_{k}$-based approaches in some nice applications [18], while Devaud et al discussed the n-degreeof-freedom oscillator case, including the possibility of varying more than one parameter of the Hamiltonian [3], without really assessing the understanding difficulties, however.

In this paper I intend to help students by approaching two aspects of the problem from elementary reasoning, using the one-dimensional harmonic oscillator as example. First I consider the answer to the recurrent question: How invariant is an adiabatic invariant? Second, in view of the fate of the single action variable $J_{k}$ of being "the" adiabatic invariant, I discuss what happens when we consider slow variations of different parameters of the oscillator, namely its spring constant $k$ and its mass $m$.

\section{How invariant is an adiabatic invariant?}

An adiabatic invariant is defined as a quantity that remains approximately constant under slow variation of a parameter $\lambda$ of an oscillating system. With slow variation we mean, not much rigorously, that being $\Delta T$ a characteristic time interval of the parametric variation and $\tau$ the period of the oscillation, then, 


$$
\Delta T>>\tau
$$

More rigorously, we can state that

$$
\frac{d \lambda}{\lambda}<<\frac{d t}{\tau}
$$

namely the fractional variation of the parameter $\lambda$ is much lower than the fraction of the period of the oscillation, defines an adiabatic process.

The conditions for the Hamiltonian $H$ to be equal the total energy $E$ are well known [5: The potential energy must be independent of the velocities and the transformation equations between vectorial and generalized coordinates must be independent of the time (see, however, 22 231). We assume that these conditions are obeyed here. Yet the condition for $H=E$ to be conserved, namely $\frac{\partial H}{\partial t}=0$, does not hold, since the parameter $\lambda$ of the Hamiltonian is time-dependent. We assume here that there is no further explicit time dependence of the Hamiltonian, that is, except for the parametric adiabatic variation, the system would be conservative.

Being $J_{k}$ the kth action variable of a multi-periodic oscillating system,

$$
J_{k}=\oint p_{k} d q_{k},
$$

where $q_{k}$ and $p_{k}$ are the canonical variables and the integral ranges over an entire cicle, textbooks [4 6] generally arrive at a formula like

$$
\dot{J}_{k}=\langle F\rangle \dot{\lambda},
$$

where $\langle F\rangle$ represents the time average of a Fourier series in which the constant term is absent, meaning that, $\langle F\rangle \simeq 0$. However, this relation does not say much, but only that the variation rate of the action variable, the adiabatic invariant $J_{k}$, is perhaps much lesser than $\lambda$. But, how much less, in quantitative terms?

In this section, let us consider a one-dimensional harmonic oscillator whose spring constant $k$ varies slowly in time, in order to answer this question. The energy of such system is no longer constant, but if the variation of the parameter is slow enough, we can consider that $\dot{E}$ and $\lambda$ hold some kind of proportionality, meaning that some relation between $E$ and $\lambda$ will remain approximately constant. Also, at each different value of $\lambda$, we assume that $E$ is constant and so the corresponding equation of motion holds. This reasoning gives rise to an alternative and more elementary, but subtle and prone to errors procedure based on the time derivative of the total energy. For the oscillator it is,

$$
\dot{E}=m \ddot{x} \ddot{x}+k \dot{x}+\frac{x^{2}}{2} \dot{k}=m \dot{x}\left(\ddot{x}+\frac{k}{m} x\right)+\frac{x^{2}}{2} \dot{k} .
$$

We can argue that the time average of the two first terms in the middle of equation (5) will vanish (for very small $k$, the adiabatic limit), since they are fast and have zero value in a period. As we isolate $\dot{x}$ from these two terms in the right-hand-side of (5), this assumption seems to find support in the equation of motion for constant $k, \ddot{x}+\frac{k}{m} x=0$, which must be obeyed for each value of $k$ (in the next section, however, we will see that what appears here as an accordance, can lead to wrong results in other situations). Yet, the term that multiplies $k$, in equation (5), $\frac{x^{2}}{2}$, a rapid term, can be replaced by its time average $\frac{\langle V\rangle}{k}$, yielding,

$$
\frac{d E}{d t} \simeq \frac{\langle V\rangle}{k} \frac{d k}{d t}=\frac{E}{2 k} \frac{d k}{d t} \quad \text { or } \quad \frac{d E}{E} \simeq \frac{d k}{2 k},
$$

where we used the well known result, $\langle V\rangle=\frac{E}{2}$ for the linear oscillator. Assuming valid the equality and solving the resulting differential equation in equation (6) we obtain,

$\frac{d}{d t} \frac{E}{\sqrt{k}}=0 \quad$ or $\quad \frac{E}{\omega}$ is the adiabatic invariant.

In fact, all linear oscilators produce the same result 18.

There is nothing new so far. However, we know that the quantity $\frac{E}{\omega}$ is not really constant. "What is the actual physical meaning of the adiabatic invariance", is a question that usually arises. To answer it let us consider an instantaneous variation $\delta \omega$ in the frequency of the oscillator, connected to $k$ by $k=m \omega^{2}$, which results in a variation $\delta E$ of the energy,

$$
\delta \omega \Rightarrow \delta E=m x^{2} \omega \delta \omega .
$$

This expression for $\delta E$ can be obtained either from equation (5) or by noting that, in the adiabatic approximation, only the potential energy is varied. Evaluating the ratio $\frac{E+\delta E}{\omega+\delta \omega}$, we obtain,

$$
\frac{E+\delta E}{\omega+\delta \omega}=\frac{E+\delta E}{\omega}\left(1+\frac{\delta \omega}{\omega}\right)^{-1} \simeq \frac{E+\delta E}{\omega}\left(1-\frac{\delta \omega}{\omega}\right),
$$

(9)

where the last passage is due to the condition $\frac{\delta \omega}{\omega}<<1$. Expanding the right-hand side of equation (9) we get,

$$
\frac{E+\delta E}{\omega+\delta \omega}=\frac{E}{\omega}+\frac{\delta E}{\omega}-\frac{E \delta \omega}{\omega^{2}}-\frac{\delta E \delta \omega}{\omega^{2}} .
$$

Now, using equation (8) above we obtain,

$$
\frac{\delta E}{\omega}=\frac{m x^{2} \omega^{2} \delta \omega}{\omega^{2}}=\frac{2 V \delta \omega}{\omega^{2}} .
$$

In a large number of cycles of the oscillator, as we replace $2 V$ by its time average $\langle 2 V\rangle=E$, we see that 
the second and third terms in relation 10 above cancel each other, leading finally to,

$$
\frac{E+\delta E}{\omega+\delta \omega}=\frac{E}{\omega}+O(\delta E \delta \omega) .
$$

Now we can understand better the concept of adiabatic invariance: a first-order variation in the slowly varying parameter $\omega$ and, in consequence, in the energy $E$, is followed by a second-order variation (a product of infinitesimals) on the ratio $\frac{E}{\omega}$, the adiabatic invariant. In other words,

\section{The time derivative of an adiabatic invariant deviates from zero only by a second-order deviation.}

\section{How many adiabatic invariants are there?}

Most advanced classical mechanics textbooks present the proof that the action variables $J_{k}$ are predestined to be the adiabatic invariants (note, however, that for more than one degree of freedom and in the presence of resonances this assumption is not trivially followed [24]). By predestined I mean that the definition of an action variable, equation (3), makes no reference to any slow parametric variation or anything like this. The number of action variables is equal to the number of degrees of freedom associated with the oscillatory motion. For the one-dimensional harmonic oscilator there is only one action variable $J$, which is easily evaluated by equation (3) and coincides with the adiabatic invariant $J=\frac{E}{\omega}$, as expected [5]. Then, a further common doubt come to students: If we consider the oscillator's mass $m$ also slowly varying in time 25, 26, how can the adiabatic invariant be the same under slow variation of different parameters? The doubts deepen when apparently correct derivations by the elementary procedure discussed in the previous section lead to results other than $J=\frac{E}{\omega}$.

I now show that common mistakes can be avoided by a procedure analogous to that of the previous section but only when we make proper use of the correct equation of motion in the derivation. Let us consider the slow variation of $m$, instead of $k$. The equation of motion is now,

$$
m \ddot{x}+\dot{m} \dot{x}+k x=0
$$

The variation rate of the energy of the oscillator becomes,

$$
\dot{E}=m \ddot{x} \ddot{x}+k \dot{x} \dot{x}+\frac{\dot{x}^{2}}{2} \dot{m} .
$$

If one makes the common (though subtle) mistake of using the same argument of the previous section, namely that the time average of the first two terms in the righthand-side vanish together, we get $\dot{E}=+\frac{\dot{x}^{2}}{2} \dot{m}$. which, after integration, leads to the supposed adiabatic invariant $E \omega$, different from the expected value $J=\frac{E}{\omega}$. What seems wrong here is that, despite the argument being valid for each term separately, we don't know for sure if they approach zero at the same rate (recall that we are dealing with variable $m$ ). So, an assumption that showed to be correct for slowly variable $k$ gives an unexpected result for slowly variable $m$. Furthermore, this result contradicts the prediction that $J$ is the only adiabatic invariant for this problem. One should note, however, that even for very small $\dot{m}$, it is not correct to take $\ddot{x}+\frac{k}{m} x=0$ anymore, which implies that the original assumption is wrong in fact, that is, the first two terms of the right-hand side of equation (14) do not vanish together. On the other hand, the equation of motion (13) for variable mass holds. Manipulating equation (14,

$$
\dot{E}=\dot{x}(m \ddot{x}+\dot{m} \dot{x}+k x)-\frac{\dot{x}^{2}}{2} \dot{m} .
$$

we see that it is the time average of the term inside parenthesis that should vanish. Accounting for this and taking the time average of $-\frac{\dot{x}^{2}}{2}$, we obtain,

$$
\frac{d E}{d t}=-\frac{\langle T\rangle}{m} \frac{d m}{d t} \quad \text { or } \quad \frac{d E}{E}=-\frac{d m}{2 m},
$$

where $T$ is the kinetic energy, which produces the correct solution,

$$
E \sqrt{m} \propto \frac{E}{\omega} \text { is the adiabatic invariant. }
$$

Finally, one can ask what happens when both parameters, $m$ and $k$ present simultaneously slow variations. Recalling that the equation of motion is still equation (13) and repeating the procedures for the separated parametric variations, we get,

$$
\frac{d E}{E}=\frac{1}{2}\left(\frac{d k}{k}-\frac{d m}{m}\right) .
$$

Now, since $\omega=\sqrt{\frac{k}{m}}$, we easily show that the righthand-side of equation 17 is equal to $\frac{d \omega}{\omega}$, so that,

$$
\frac{d E}{E}=\frac{d \omega}{\omega},
$$

which is equivalent to say that, again, $\frac{E}{\omega}$ is the adiabatic invariant.

\section{Conclusions}

The identification of the action variables with the adiabatic invariants of an oscillatory systems does not help students to understand the very concept of adiabatic invariance. I hope I have clarified in section 2 the actual meaning of adiabatic invariance in more understandable terms. The elementary method based on time derivative of the energy, on the other hand, is subject to subtle mistakes that can yield different results when different 
parameters are considered to vary slowly. I intended to show in section 3 , based on a simple example, that the two approaches yield the same correct result when the proper equation of motion is resorted to. These two points are surely included in any formal derivation based on action variables such as that in ref. [11 but it appears that the present alternative approach can help students to better understand the concept of adiabatic invariance in classical mechanics.

\section{Acknowledgements}

The author enjoyed numerous discussions with graduate students in classes of Analytical Mechanics and with fellows of the Physics Department of the Universidade Federal de Minas Gerais. This work had support from Conselho Nacional de Pesquisas, CNPq (Brazilian agency).

\section{References}

[1] R. Talman, Action/Angle variables ans adiabatic invariance, available in http://uspas.fnal.gov/materials/02Yale/AdiabaticInv.pdf.

[2] J.J. Sakurai, Modern Quantum Mechanics (AddisonWesley, Reading, 1994).

[3] M. Devaud, V. Leroy, J. Bacri and T. Hocquet, Eur. J. Phys. 29, 831 (2008).

[4] L. Landau and E. Lifschitz, Mechanics (MIR, Moscow, 1966).

[5] H. Goldstein, Classical Mechanics (Addison-Wesley, Reading, 1980).

[6] J.V. José and E.J. Saletan, Classical Dynamics (Cambridge University Press, Cambridge, 1998), p. 71.

[7] K.R. Symon, Mechanics (Addison-Wesley, Reading, 1972), p. 379

[8] S.T. Thornton and J.B. Marion, Classical Dynamics of Particles and Systems (Cengage Learning, Boston, 2004), p. $258-261$.

[9] G.R. Fowles and G.L. Cassiday, Analytical Mechanics (Cengage Learning, Boston, 2005), p. 455.

[10] N.A. Lemos, Mecânica Analítica (Livraria de Física, São Paulo, 2007) p. 308-309.

[11] D.E. Neuenschwander and S.R. Starkey, Am. J. Phys. 61, 1008 (1993).

[12] C.S. Gardner, Phys. Rev. 115, 791 (1959).

[13] L. Parker, Am. J. Phys. 39, 24 (1971).

[14] M.G. Calkin, Am. J. Phys. 45, 301 (1977).

[15] A.M. Cetto and L. de la Peña, Am. J. Phys. 52, 539 (1984).

[16] J. Siverdière, Eur. J. Phys. 9, 150 (1988)

[17] C. Gignoux and F. Brut, Am. J. Phys. 57, 422 (1989).

[18] F.S. Crawford, Am. J. Phys. 58, 337 (1990).

[19] C.G. Wells and T.C. Siklos, Eur. J. Phys. 28, 105 (2007).

[20] L.L. Sanchez-Soto, Am. J. Phys. 81, 57 (2013).

[21] R. Borghi, Eur. J. Phys. 34, 4221287 (2013).

[22] J.R. Mohallem, Am. J Phys. 83, 857 (2015).

[23] J.R. Mohallem, As entrelinhas da mecânica Lagrangiana e Hamiltoniana (Livraria da Física, São Paulo, 2017) p. 45-48.
[24] V.I. Arnold, V.V. Kozlov and A.I. Neishtadt, Mathematical Aspects of Classical and Celestial Mechanics, (Springer, Heidelberg, 2006) 3rd ed., p. 324.

[25] E. Correa, J.S. Espinoza Ortiz, M. Valério and J. Dutra, Rev. Bras. Ensino Fís. 33, 4307 (2011).

[26] H. Rodrigues, N. Panza and D. Portes Jr., Rev. Mex. Fis. 60, 31 (2014). 